\title{
Prevenção Quaternária: da medicalização social à atenção integral na Atenção Primária à Saúde
}

\author{
Quaternary Prevention: from social medicalization to integral care on the Primary Health Care \\ Prevención Cuaternaria: de la medicalización social a la atención integral en Atención Primaria de \\ Salud
}

\begin{abstract}
Karina Schopf ${ }^{1}$ (1)
Carine Vendruscolo ${ }^{1}$ (D)

Clarissa Bohrer da Silva² (1)

Daniela Savi Geremia ${ }^{3}$ (1)

Aline Lemes de Souza ${ }^{1}$ (B)

Lucas Lasta Angonese ${ }^{2}$ (d)
\end{abstract}

1. Universidade do Estado de Santa Catarina,

Programa de Mestrado Profissional em

Enfermagem na Atenção Primária à Saúde.

Chapecó, SC, Brasil.

2. Universidade do Estado de Santa Catarina. Chapecó, SC, Brasil.

3. Universidade Federal da Fronteira Sul.

Chapecó, SC, Brasil.
Autor correspondente:

Karina Schopf.

E-mail: karinaschopf70@gmail.com.

Recebido em 25/05/2021.

Aprovado em 17/08/2021.

\begin{abstract}
Resumo
Objetivo: conhecer as percepções e perspectivas dos profissionais da saúde para o desenvolvimento da Prevenção Quaternária na Atenção Primária à Saúde. Método: Pesquisa Apreciativa, que aplicou as fases do "ciclo 4-D", na língua inglesa: discovery dream, design e destiny. Este artigo analisa os resultados referentes à fase discovery (descoberta), de cujos dois encontros correspondentes participaram nove profissionais da Atenção Primária. Realizou-se análise de conteúdo, seguindo as etapas de pré-análise, exploração do material e tratamento dos dados. Resultados: a Prevenção Quaternária representa uma potencialidade na inovação da atenção, com possibilidade de reduzir a medicalização social, que ocorre mediante a sobremedicalização, sobrediagnósticos e sobretratamentos. Como perspectivas, é apresentada a necessidade de conscientização da sociedade e dos profissionais sobre esses excessos e de (re) posicionamento da indústria, do mercado e da mídia sobre o significado de "estar saudável". Conclusão e implicações para prática: é necessário atentar para a ética na prestação de cuidados quanto ao rastreio, diagnóstico e tratamento de doenças. A Prevenção Quaternária tem potencial para reverter um modelo hegemônico em relação ao cuidado de indivíduos e famílias ao fomentar a integralidade. A Enfermagem, como prestadora do cuidado, junto com a equipe multiprofissional, deve incorporar ações de Prevenção Quaternária em suas práticas.
\end{abstract}

Palavras-chave: Atenção Primária à Saúde; Educação Continuada; Enfermagem; Medicalização; Prevenção Quaternária.

\section{Abstract}

Objective: to know the perceptions and perspectives of health professionals for the development of Quaternary Prevention in Primary Health Care. Method: an Appreciative Research, which applied the phases of the "4-D cycle": discovery, dream, design and destiny. This article analyzes the results referring to the discovery phase (discovery), in which nine professionals from Primary Care participated in two corresponding meetings. Content analysis was performed, following the steps of pre-analysis, material exploration and data treatment. Results: the Quaternary Prevention represents a potentiality in care innovation, with the possibility of reducing social medicalization, which occurs through over-medicalization, over-diagnosis and overtreatment. As perspectives, it is presented the need for society and professionals to be aware of these excesses and the (re) positioning of the industry, market and media about the meaning of "being healthy". Conclusion and implications for the practice: it is necessary to pay attention to ethics in care provision regarding screening, diagnosis and treatment of diseases. Quaternary Prevention has potential to revert a hegemonic model in relation to the care of individuals and families by promoting integrality. Nursing as a care provider, together with the multi-professional team, must incorporate Quaternary Prevention actions in its practices.

Keywords: Continuing Education; Medicalization; Nursing; Primary Health Care; Quaternary Prevention.

\section{Resumen}

Objetivo: conocer las percepciones y perspectivas de los profesionales de la salud para el desarrollo de la Prevención Cuaternaria en Atención Primaria de Salud. Método: Investigación Apreciativa, que aplicó las fases del "ciclo 4-D", en inglés: discovery, dream, design y destiny Este artículo analiza los resultados de la fase discovery (descubrimiento), en cuyos dos encuentros correspondientes participaron nueve profesionales de Atención Primaria. Se realizó análisis de contenido, siguiendo los pasos de pre-análisis, exploración de material y procesamiento de datos. Resultados: la Prevención Cuaternaria representa un potencial en la innovación asistencial, con posibilidad de reducir la medicalización social, que se produce por sobremedicalización sobrediagnóstico y sobretratamiento. Como perspectivas, se presenta la necesidad de concienciar a la sociedad y los profesionales sobre estos excesos y de (re) posicionar a la industria, el mercado y los medios de comunicación sobre el significado de "estar sano". Conclusión e implicaciones para la práctica: es necesario prestar atención a la ética en la prestación de cuidados en cuanto al cribado, diagnóstico y tratamiento de enfermedades. La Prevención Cuaternaria tiene el potencial de revertir un modelo hegemónico en relación al cuidado de las personas y familias al promover la integralidad. La Enfermería, como proveedora de cuidados, junto con el equipo multidisciplinario, deben incorporar las acciones de Prevención Cuaternaria en sus prácticas.

Palabras clave: Atención primaria de salud; Educación continua; Medicina social; Medicalización; Prevención cuaternaria. 


\section{INTRODUÇÃO}

Os determinantes de saúde podem envolver fatores sociais, culturais, políticos, econômicos, ambientais, comportamentais e biológicos ${ }^{1,2}$. Reconhecer a influência desses fatores é essencial para planejar ações de saúde pública, atendendo ao princípio de integralidade, com base na promoção da saúde e na prevenção de doenças. No âmbito da prevenção, a dinâmica psicossocial, econômica e cultural é fundamental e implica a efetivação da Prevenção Quaternária $(\mathrm{P} 4)^{3}$.

A P4 é proposta no contexto dos três níveis clássicos de prevenção ${ }^{4}$, compreendida como um quarto nível, que reverbera em mudanças na forma de realizar a prática clínica, protegendo o indivíduo do excesso de intervenção em saúde, como a medicalização e os rastreamentos, que culminam em sobrediagnósticos e sobretratamentos e, de certa forma, de outros interesses lucrativos decorrentes da mercantilização da saúde. Nesse sentido, é necessário refletir acerca da influência das ditas "indústrias da saúde" sobre os atores que colaboram na sua produção, sejam eles profissionais, gestores públicos ou comunidade 5 .

A medicalização vinculada à dependência de intervenções profissionais reduz a autonomia do indivíduo quando o seu estado de saúde está relacionado a situações de sofrimento, adoecimento, perdas e lutos, ocasionando o aumento das intervenções e prejuízos sociais ${ }^{6}$. Cabe, aos profissionais de saúde, identificar ações e manejar as demandas visando a evitar a medicalização social nos serviços da Atenção Primária à Saúde (APS) ${ }^{7}$.

A P4 envolve a responsabilidade do profissional de saúde em identificar pacientes/usuários que estejam em risco de práticas intervencionistas desnecessárias, as quais podem causar mais danos do que benefícios à saúde, zelando por esses indivíduos ${ }^{8}$. Para tanto, os princípios éticos e morais de proteção aos usuários necessitam prevalecer diante do risco de agravos causados pelos excessos, especialmente quando se trata de pessoas saudáveis.

As intervenções clínicas e medicamentosas, muitas vezes, são endossadas pelas falsas promessas de longevidade, sendo negados o direito e a capacidade da pessoa em lidar com os infortúnios voltados à sua saúde ${ }^{9}$. Diante destes, a necessidade de um diagnóstico clínico faz com que os indivíduos busquem, contínua e desnecessariamente, os serviços de saúde, o que gera o consumo de tecnologias médicas e, eventualmente, transforma fatores de risco em doença. Para os profissionais de saúde, a imposição da sociedade em chegar a um diagnóstico acarreta sobrediagnósticos e sobretratamentos que, na maioria das vezes, não trazem benefícios ao usuário e, ainda, podem acarretar danos em curto, médio ou longo prazo ${ }^{10}$.

Cumpre destacar que pequenas alterações nos limites de diagnósticos influenciam diretamente a saúde, levam a diagnósticos e tratamentos exagerados, muitas vezes, em pessoas assintomáticas, que são diagnosticadas e tratadas com o uso de insumos tecnológicos e profissionais, ancorados por influências culturais, estreitando o limiar do que é saúde e doença ${ }^{2}$. Soma-se a isso a implementação de protocolos e campanhas sanitárias, por vezes, distorcidos, que deslumbram a população com a facilidade de prevenir e tratar doenças, ocasionando a falsa sensação de proteção.

Os excessos de exames e diagnósticos associados à produção de saúde evidenciam paradigmas conflitantes em que a tomada de decisão dos profissionais pode pecar pela demasia em detrimento da falta. Esse tipo de atitude profissional, acarretado pela cultura biomedicista e permeado pelo medo e incerteza, deixa os profissionais de saúde desconfortáveis em relação à P4. Além disso, as pessoas procuram melhorar a qualidade de vida por meio de intervenções em saúde, por acreditar que essas podem resolver possíveis adversidades, corroborando uma medicina defensiva e paternalista, que favorece as indústrias da saúde, o consumismo e as intervenções desnecessárias ${ }^{11}$.

Assim, o diálogo sobre a P4 extrapola a área médica e exige a atuação dos diferentes profissionais de saúde, especialmente os prescritores. A APS é considerada o berço da P4 por ser o campo propício para o seu desenvolvimento por meio da escuta qualificada, vínculo e adaptação individualizada dos atendimentos em saúde. Além disso, a P4 vai ao encontro dos princípios preconizados na Estratégia Saúde da Família (ESF) ${ }^{12}$ ao incentivar a interprofissionalidade. Esta organização do trabalho de equipe auxilia a evitar possíveis iatrogenias, causadas pelo excesso de intervenções clínicas, como rastreamentos e sobremedicalização, por meio do trabalho colaborativo das equipes de Saúde da Família (eSF), equipes de Saúde Bucal (eSB) e Núcleos Ampliados de Saúde da Família e Atenção Básica (Nasf-AB).

Nessa perspectiva, esta pesquisa teve como objetivo conhecer as percepções e perspectivas dos profissionais de saúde para o desenvolvimento da P4 na APS.

\section{MÉTODO}

Trata-se de um estudo de abordagem qualitativa, com base na Pesquisa Apreciativa (PA), a qual visa à transformação da realidade individual e em equipe, vivenciada pelos participantes, por meio dos pontos fortes, de experiências positivas e de ideias novas $^{13}$. Dentre as possibilidades oferecidas pela PA, estão: transformar os problemas em oportunidades; identificar os pontos positivos dos participantes e da instituição; criar possibilidades; diminuir as críticas e possibilitar o crescimento com base na força e nas experiências exitosas ${ }^{14}$.

O "ciclo 4-D" da PA consiste em quatro pilares cuja denominação foi formulada a partir da língua inglesa: discovery (descoberta) - no qual o grupo identifica os pontos fortes e as competências essenciais; dream (sonho) - em que, juntos, imaginam possibilidades positivas para o futuro; design (planejamento) - no qual há a projeção de aspirações para cada oportunidade e, por fim, destiny (destino) - em que o grupo elabora o processo e as estruturas para alcançá-los ${ }^{15}$ (Figura 1).

A pesquisa foi realizada com nove profissionais atuantes na APS, pertencentes a uma equipe de eSF, uma eSB e uma de Nasf-AB, em um município do Extremo Oeste de Santa 


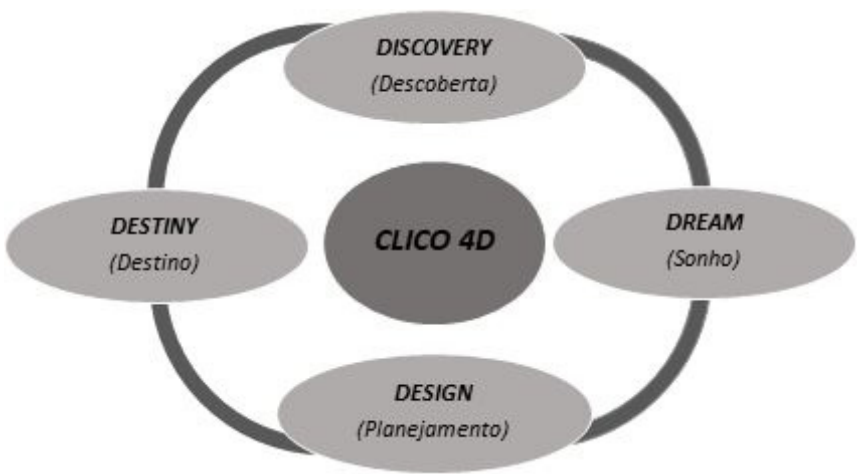

Figura 1. Apresenta o "ciclo 4D" da Pesquisa Apreciativa ${ }^{15}$. Fonte: Adaptada pelos autores, 2020.

Catarina. Foi critério para a inclusão dos participantes da pesquisa: ser profissional de nível superior, atuante em uma das equipes mencionadas, por dois anos ou mais. Excluíramse os participantes que, por algum motivo, estavam afastados no período da produção das informações. Participaram nove profissionais, sendo eles: enfermeiro (um); médico (um); dentista (um); farmacêutico (um); psicólogo (dois); fisioterapeuta (dois); nutricionista (um).

Ao todo, a pesquisa contou com cinco encontros, contemplando as quatro fases do ciclo 4-D. A fase da descoberta foi organizada em dois encontros e teve como objetivo conhecer a percepção dos profissionais sobre a $\mathrm{P} 4$ e levantar os pontos fortes, habilidades pessoais e institucionais para o desenvolvimento de ações. O terceiro encontro foi a fase do sonho com o objetivo de imaginar o melhor ideário de ações voltadas à $\mathrm{P} 4$ que podem ser realizadas no cotidiano da equipe. $O$ quarto encontro, referente ao planejamento, objetivou criar possibilidades para ações de P4 com base na realidade da equipe e da instituição. Por fim, o quinto e último encontro do ciclo 4D - o destino - foi aquele no qual os participantes realizaram a pactuação das ações de $\mathrm{P} 4$ escolhidas pela equipe em uma matriz de intervenções.

Todos os encontros foram realizados no mês de julho de 2020, com intervalos aproximados de sete dias e duração média de uma hora e quinze minutos cada. A participação média de cada encontro foi de seis profissionais. A organização dos encontros foi definida de acordo com o rigor metodológico da $\mathrm{PA}$; já os diálogos foram construídos pelo grupo e norteados por questões disparadoras voltadas ao tema apreciado.

Uma sequência lógica foi aplicada aos encontros, garantindo tempo apropriado e a efetiva participação do grupo nos diálogos. No início de cada um, utilizou-se um momento de "aquecimento" a fim de interagir e envolver os participantes na pesquisa. Ao final dos três primeiros encontros, cada participante recebeu uma atividade reflexiva para realizar individualmente até o encontro seguinte, sendo retomada e discutida no grupo. A atividade reflexiva consiste em viabilizar a reflexão acerca do objeto do estudo e contribui na estratégia para preparar o encontro seguinte ${ }^{15}$.
Neste artigo, serão analisados e discutidos os resultados do primeiro e segundo encontros da PA, que corresponderam à fase da descoberta. Os encontros foram gravados em áudio e, posteriormente, transcritos mediante o consentimento dos participantes. Além disso, houve o registro dos temas principais, que emergiram durante os diálogos, sob a forma de um diário de campo produzido pelos pesquisadores com o intuito de facilitar a transcrição e análise dos dados.

Para o tratamento das informações, foi utilizada a Análise Temática de Conteúdo, ${ }^{16}$ seguindo os momentos de pré-análise, exploração do material e tratamento dos dados. A pré-análise consistiu na organização dos depoimentos transcritos mediante a retomada dos objetivos iniciais da pesquisa. Foram realizadas leituras e releituras de todo o material oriundo dos encontros (transcrições dos diálogos e anotações em diário) e a organização dos relatos, objetivando ter uma visão geral do que foi dito por eles, percebendo as particularidades. A exploração do material permitiu apreender a relevância das falas de cada participante, classificar as ideias centrais e organizá-las em duas categorias: Percepções dos profissionais sobre P4 e Perspectivas para o desenvolvimento de ações de P4 na APS.

Cumpre destacar que, durante a realização dos encontros, diferentes reflexões e ideias surgiram dos diálogos, algumas ao encontro dos autores referenciados nas atividades reflexivas e outras não, as quais influenciam, direta ou indiretamente, a percepção sobre o conceito e as possíveis práticas da P4 que poderiam ser adotadas pela equipe. Contudo, o ideário, que orienta pesquisas participativas, pressupõe essa construção coletiva e o propósito é a transformação da realidade. ${ }^{15}$

A pesquisa foi aprovada pelo Comitê de Ética em Pesquisa com Seres Humanos sob o Protocolo número 3.375.951 de 6 de junho de 2019. Para garantir o anonimato das participantes, foram utilizados os seguintes códigos: a letra inicial da categoria profissional seguida de um número sequencial no caso de haver mais de um profissional da mesma categoria.

\section{RESULTADOS}

\section{Percepções dos profissionais sobre P4}

A partir da realidade e experiência no serviço, quanto à percepção em relação à $\mathrm{P} 4$, os profissionais demonstraram apreensão e dificuldade em reconhecer a P4 nas ações práticas. Os participantes do estudo alertam para um ideário dos usuários marcado pela centralidade na doença e na cura, por meio da medicação, além da pressão exercida pelos meios de comunicação e agentes políticos, que influenciam a população e atrapalham a realização de ações de $\mathrm{P} 4$.

[...] a mídia auxilia muito na questão do estar doente, do querer ser doente, que já traz auxílio para alguma coisa que você nem tenha [...] o usuário já vem consultar sabendo o que ele vai querer, às vezes, sem indicação médica ou orientação [...] (FO1). 
[...] judicialização [...] não atenção devida ao quadro que, futuramente, pode estar se instalando [...] é o que, muitas vezes, induz aos profissionais quererem abranger todas as possibilidades que existem de se estabelecer um diagnóstico ou, pelo menos, para descartar um possivel (D1).

[...][os usuários] já vêm com a relação de medicação e exames, principalmente os mais complexos, na maioria das vezes, nem condizentes com os sintomas que ele está apresentando [...] (M1).

[...] A gente vê que é muito mais trabalhoso tentar desmistificar a necessidade dos exames [...] a própria instituição por meio de alguns agentes; você é cobrado na tentativa de favorecer o gosto do paciente e não exatamente da técnica e da parte científica ou da conduta correta que deveria [...] então, o profissional acaba sendo induzido [...] (E1).

Dentre as ações e práticas assistencialistas, que são identificadas pela equipe e que podem causar mais danos do que benefícios à saúde dos usuários, estão a realização indiscriminada de exames e medicações. Sob a ótica dos profissionais, evidenciase a manifestação de crenças dos usuários de que o exame, por si só, seja a solução, já que revela a doença. Nesse ínterim, a falta de conscientização dos usuários influencia o controle de algumas patologias, como a dislipidemia, regulada com o uso indiscriminado de medicação em detrimento da dieta e de outros hábitos saudáveis. Os profissionais de saúde, em seus depoimentos, esclarecem como isso acontece.

[...]as mamografias com solicitação indiscriminada. Deveria ser realizado exame físico e interpretação individual para ser solicitado, lembrando que, dentro do rastreamento, a maioria das mamografias já vem com a solicitação de ultrassonografia (E1).

[...] as doenças crônicas que retornam muito: paciente de 80 anos com artrose faz $R X$, faz fisioterapia, se não melhorar, vamos pedir uma ressonância. Então, ela já vem com a ideia que não vai melhorar e que precisa de uma ressonância (FA1).

[...] eu até iria mais a fundo, poucos relacionam o exame com a descoberta da doença, muitas vezes, eles esperam que, com o exame, eles vão melhorar (E1).

[...] outro fator vivenciado pela nutricionista é o controle de dislipidemia com medicação por pacientes que não se conscientizam [uso indiscriminado de medicação] (E1).

Fatores externos relacionados a situações socioeconômicas e familiares também são identificados como determinantes para o excesso de intervenções realizadas pela equipe, com destaque para a alta demanda de exames na confirmação de diagnósticos que geram afastamento do trabalho (atestados médicos). Isso, aliado aos que parecem encontrar alento e até forçar situações de doença, compreende questões que geram preocupação na equipe de saúde.

[...] a busca por atestados no INSS [Instituto Nacional do Seguro Social] também é uma demanda que gera exames (FA1).

[...] podemos fazer um estudo, mas, certamente, 30 a $40 \%$ dos atendimentos giram em torno desses atendimentos [INSS] (E1).

[...] às vezes, vejo que a atenção que eles desejam em casa eles não têm, então, o fator doença, pra eles, é algo bom, principalmente os idosos [...] (FA1).

\section{Perspectivas para o desenvolvimento de ações de P4}

Apesar dos desafios apresentados nas percepções dos profissionais sobre a temática e as relações com os usuários, os profissionais mapearam algumas possibilidades para 0 desenvolvimento de práticas de $\mathrm{P} 4$ no serviço, que passam por um atendimento integral pautado na escuta ativa e anamnese detalhada do usuário, além de uma atuação em equipe de forma compartilhada.

[...] uma boa anamnese, conversa para entender o que o paciente está procurando, muitas vezes, o profissional já vai fazendo a conduta antes dele terminar de falar o que ele quer, já vai prescrevendo exames e o paciente vai embora. Inicia-se com uma boa anamnese (FA1).

[...] tempo para atendimento, isso é imprescindível, a partir de que você não tem tempo, tudo isso aqui éjogado fora, tempo para uma boa anamnese, olhar prontuário, ver se ele precisa dessa medicação, se é necessário mudar medicação, então, tudo isso demanda muito tempo [...] (M1).

[...] a realidade é que minha postura é atender paciente, não tem uma postura de equipe [...] atendimento compartilhado (M1).

[...]o médico, às vezes, pode encaminhar, primeiro, pra fisio [Fisioterapeuta] antes de fazer algum exame, eu acho uma tentativa legal (FA1).

Os profissionais também relatam mudanças de atitudes dos usuários, os quais já chegam na Unidade Básica de Saúde (UBS) com demandas específicas e, por vezes, voltadas à resolução momentânea de problemas ou com expectativas e intenções medicalizantes. Assim, reconhecem a necessidade de estudos de análise epidemiológica do território, a realização da educação em saúde para o envolvimento do usuário na produção da saúde e para sensibilizar no desenvolvimento das práticas de P4. 
[...] a longo prazo, eles não querem resolver, eles querem uma medicação para aliviar a dor momentânea (FO1).

[...] esse problema de INSS é muito grande[...] para o paciente que não há necessidade daquilo que ele está procurando, tu vês, no olho dele, uma decepção porque ele está entendendo que corre o risco de não passar e ganhar aquilo que ele quer (M1).

[...] falar com propriedade como realmente está nossa população nas suas necessidades reais, nós não temos esse estudo [levantamento e análise de dados epidemiológicos do território] (E1).

[...] o paciente já vem com a cabeça formada do que ele quer, ele acha que vai ser perda de tempo ele participar de uma palestra, de uma [ação de] educação em saúde (P2).

[...] a população acaba frustrando, percebem que eles têm que fazer, que eles têm que tomar atitude, que não tem um pó mágico (P1).

[...] se o profissional tira o que o paciente quer, passa por ruim (D1).

Foram destacadas as Práticas Integrativas e Complementares (PIC) em saúde como uma alternativa de cuidado, em contraponto à medicalização, considerando a necessidade de uma "resposta" para o problema apresentado pelo usuário. Estas configuram-se como elemento potencial, que contribui para a aplicabilidade da P4.

[...]eu vejo que, a partir de agora, as coisas têm a melhorar com as práticas integrativas nas Unidades de Saúde. Os pacientes, além da consulta, às vezes, vêm só para essas práticas e nem voltam para o médico [...] aqui, o pessoal faz auriculoterapia, gente que relatou na farmácia, para mim, que passou a dor e que vai continuar com a aurículo, não vai precisar mais tomar remédio, é um caminho que veio para auxiliar a desmedicalização e o excesso de intervenções[...] (F01).

Contudo, apesar de reconhecerem as concepções "médico centradas" e medicalizantes por parte do usuário, bem como as atitudes políticas dos gestores, os profissionais de saúde acreditam que os movimentos de mudança devem partir deles próprios.

[...] Existe o desejo da população e a administração sempre vai tentar fazer o que pode pela administração [questões políticas]. Muitas vezes, se ela [administração] não está ligada à parte técnica dos profissionais, ocorre esse fluxo desordenado, nada resolutivo. Vai dar as 18 fichas todos os dias, vai dar acúmulo de exames. Tem demanda mapeada para tanto tempo, é sempre a mesma parcela da população, essa é a questão (E1).

[...] acho que tem mais a ver mesmo com a questão do profissional do que da população [o profissional ter postura voltada à P4] (FA2).
Os participantes destacam que a mudança demanda tempo, conhecimento, planejamento e, em certa medida, motivação. O cotidiano é repleto de rotinas e demandas, havendo pouco tempo e incentivo para atualizações técnicas e científicas relacionadas às práticas de atenção à saúde e, principalmente, à P4.

[...]a maioria das pessoas pensa que a gente vai fazer um trabalho aqui de hoje para amanhã, é muita descoberta, é muita discussão, [...] gente vai ter que sair do que 'eu acho' para o que 'nós achamos', essa situação que é o maior desafio [...]motivação é tudo [...] (E1).

[...] precisamos ter um momento para discutir: "Dia tal vai ter reunião para fazer isso... um cronograma" (M1).

[...] essa motivação, muitas vezes, a gente vai buscar o conhecimento fora [...] às vezes, nós temos que tirar do nosso bolso e ainda vai ter resistência da administração que você terá que se ausentar por um período, e não é perceptível aos olhos de muitos que isso vai trazer benefícios para a população [...] (D1).

[...] a gente não se sente abraçado por todos [equipe e gestão], é tudo muito individual (P2).

\section{DISCUSSÕES}

Ao considerar a importância do tema para a saúde integral, a P4 precisa ser realizada de forma fracionada ante aos demais níveis de prevenção (primária, secundária e terciária) ${ }^{4}$ nos âmbitos estrutural, particular e singular da organização e funcionamento dos serviços de saúde. Isso colabora para evitar danos desnecessários à saúde do usuário e demanda a utilização de um ideário que extrapole o modelo biomédico e que individualize a prevenção 8,17 mediante posicionamentos profissionais éticos e menos corporativistas.

O apelo à prevenção e à promoção da saúde, geralmente, recorre aos saberes biomédicos que, em sua essência, conduzem práticas de autocuidado especializado, cujo potencial de promoção de autonomia e desmedicalização é limitado, já que os usuários são dependentes do saber alheio e remetidos à adesão ao que é cientificamente recomendado, com base em estatísticas de grupos populacionais, mas que, por vezes, transforma pessoas saudáveis em doentes ${ }^{17}$.

É preciso repensar a organização dos serviços de saúde, rever a postura profissional, mas, também, as práticas de comunicação com os usuários de tal forma que a relação médico-usuário se amplie para uma relação profissional-usuário, sendo este último médico ou outro. Os achados iniciais desta pesquisa demonstram que os profissionais tendem a responsabilizar outros seguimentos quanto às (ausência de) práticas de $\mathrm{P} 4$, como, por exemplo, a mídia, os próprios usuários e o gestor de saúde. Contudo, ao longo dos diálogos, reconhecem que a grande transformação das práticas de cuidado e a inserção das ações de P4 podem ser implementadas e fortalecidas a partir da compreensão e 
iniciativa da própria equipe de saúde. Isso é importante, pois, quando se aproximam diferentes disciplinas e competências profissionais em um mesmo objetivo, como a saúde pública/ coletiva e o foco na APS, há pontos de cruzamento entre elas, indicando um campo de saber e de prática que é comum e que demanda habilidades e competências dos profissionais para 0 trabalho em equipe, contribuindo para a qualidade na atenção à saúde ${ }^{18}$. Assim, operar, de forma interprofissional, implica interagir com áreas/profissionais de diversas formações/núcleos de saber e cujas práticas envolvem um "saber comum", o qual transita entre áreas específicas, de maneira complementar e colaborativa, com vistas à produção de saúde ${ }^{19}$.

As percepções dos participantes, referentes à contribuição dos usuários como entrave no desenvolvimento de ações voltadas à $\mathrm{P} 4$, podem ser entendidas e analisadas em relação à responsabilização dada ao sistema de saúde frente às iniquidades sociais, às influências culturais e econômicas, incluindo a busca pela saúde a qualquer custo e, de preferência, sem sacrifícios ou envolvimento, que, de forma subjetiva, acarreta diversos problemas de saúde ${ }^{20}$. É importante ressaltar que essa inversão de valores, por vezes, prejudica o acesso daqueles que precisam e amplia as intervenções para aspectos comuns da vida, tornando $o$ atendimento reducionista, fragmentado e vicioso, dispensando recursos e atenção com pessoas saudáveis em detrimento daquelas que realmente necessitam de atendimento ${ }^{21}$.

Ao considerar o papel do marketing aplicado a situações de saúde, como a excessiva ênfase em aspectos curativos e também "preventivos", que emergem da grande procura pelos serviços de saúde, há risco de transformar eventos vitais em patológicos e, nesse sentido, algumas mudanças podem contribuir, como: entender que saúde não é marketing, não é mercadoria e que todos os condicionantes e determinantes sociais, políticos e profissionais devem ser analisados para o melhor atendimento prestado $^{20}$.

Nessa linha, a pressão exercida pelas indústrias, mercados e marketing da saúde, com abordagens biologicistas, é altamente disseminada nas mídias e na sociedade. Alguns desses desafios foram identificados pelos participantes para a efetivação da P4. A doença sempre foi vista como lucrativa, mas, atualmente, mais do que nunca, os não doentes são alvo desse mercado, que interfere significativamente na postura e prática assistencialista dos profissionais de saúde ${ }^{22}$. Notoriamente, ações como a medicalização social interferem na competência do manejo das adversidades da saúde pelos indivíduos, dificultando a sua compreensão de situações voltadas ao adoecimento, sofrimento emocional, morte e outras situações inerentes à vida humana ${ }^{9}$.

Por conseguinte, é necessário consolidar o conceito e efetivar práticas de $\mathrm{P} 4$ na relação que se estabelece entre os profissionais e a população, utilizar as redes sociais, mídias de comunicação, líderes comunitários e momentos de educação em saúde nas escolas e em outros locais na comunidade, visando ao empoderamento dos indivíduos quanto à saúde ${ }^{23}$. Nessa direção, as políticas públicas, ao pautarem a P4 como um dos princípios norteadores da qualificação das eSF como ferramenta da APS, poderão contribuir para minimizar tratamentos farmacológicos, exames complementares e rastreamentos desnecessários, evitando danos aos usuários, com vistas ao cuidado clínico-sanitário.

O reconhecimento, por parte de alguns profissionais participantes da pesquisa, sobre a sua responsabilidade profissional na $\mathrm{P} 4$ vai ao encontro da premissa ética de primeiro "não fazer"24. Concomitante a isso, pode-se pensar em práticas que envolvam e orientem os usuários, fortalecendo vínculos de confiança, de troca e de satisfação em que ambos possam ser conhecedores dos riscos e dos benefícios do excesso de intervenções, possibilitando uma relação de corresponsabilidade e não de culpa, como é de receio dos profissionais. Nessa conjuntura, práticas que reduzem a medicalização e a iatrogenia do cuidado devem configurar-se como indutoras de novas subjetividades e práticas de saúde.

Além dos fatores já relatados, os profissionais destacaram as demandas, que, em excesso, diminuem o tempo dispensado para cada usuário e, como consequência, aumentam as intervenções clínicas e a medicalização, dificultando a relação profissionalusuário, favorecendo as intervenções, como os rastreamentos (screening), que, quando não organizados, são potencialmente prejudiciais à saúde do indivíduo. Nessa premissa, é obrigação do profissional garantir atendimento integral, valorizar a escuta qualificada e o conhecimento técnico descomprometido de influências das indústrias da saúde ${ }^{25}$.

Entretanto, é consensual que o excesso de demandas dos usuários na APS causa angústia e frustração nos profissionais de saúde, seja pela dificuldade em entender ou auxiliar o indivíduo em suas necessidades, seja por questões socioeconômicas, de infraestrutura ou mesmo por condições de saúde nas quais nada pode ser feito para minimizar a dor e o sofrimento. Em alguns casos, busca-se oferecer o conforto por meio da medicalização e intervenções desnecessárias como forma de resolver rapidamente a condição apresentada ${ }^{26}$. Cabe destacar que o dimensionamento de pessoal inapropriado, muito comum nas eSF, favorece o aumento da demanda e, consequentemente, da medicalização e intervenções ${ }^{21}$.

Ao considerar a demanda excessiva por atendimentos que, por vezes, prejudicam a realização e, consequentemente, a qualidade da anamnese, esta é apresentada, pelos participantes, como fundamental para a concretização da P4 e deve ser completa, priorizada não só nas consultas médicas, mas, também, nas consultas de Enfermagem no intuito de reduzir intervenções e a medicalização. Neste cenário, faz-se necessário ampliar os momentos de escuta qualificada como ferramenta que tem potencial para contribuir na identificação das necessidades do usuário e, consequentemente, auxiliar na anamnese e interpretação dos problemas apresentados, possibilitando a construção do plano de cuidado individualizado ${ }^{27}$.

Os participantes apontam, como perspectiva, o atendimento de saúde compartilhado, que pode ser alternativa para o excesso de demanda e garantia do acesso, assim como para a redução das intervenções desnecessárias. Estes poderão usufruir de 
Projeto Terapêuticos Singulares (PTS) e de outras ferramentas que possibilitem a prática do processo de trabalho articulado e interdisciplinar. Destaca-se que mais profissionais estarão envolvidos e serão conhecedores da situação de saúde daquele usuário, o que possibilitará atendimento integral e oportuno, que deve estar presente na APS ${ }^{28}$.

Para os participantes da pesquisa, a atividade clínica precisa ser embasada em aspectos epidemiológicos e que permitam identificar e agir de acordo com a realidade da população atendida. É preciso atenção dos profissionais para não reduzir o cuidado a protocolos e intervenções baseados em diretrizes que não valorizam a escuta qualificada e as particularidades dos indivíduos ou famílias ${ }^{21}$.

A oferta de PICS, como recurso de manejo da medicalização, porta a indefinição de poder significar estímulo ao autocuidado especializado, mas, também, ao autorreferido. Sua empregabilidade tem um positivo potencial desmedicalizador, que pode se tornar uma estratégia de redução de danos ${ }^{5}$. Portanto, as PIC podem representar uma alternativa justificável em situações nas quais as intervenções biomédicas significam riscos adicionais e poucos benefícios potenciais.

Os participantes apontaram o posicionamento dos gestores, por vezes, político, com interesses exclusivos de agradar à população sem conhecer os aspectos técnicos e científicos que subsidiam as ações de saúde voltadas à $\mathrm{P} 4$, além da rotatividade dos gestores e o não conhecimento sobre o tema apreciado, ancorados pela falta de incentivo para as ações de educação permanente, e o desconhecimento da Política Nacional de Educação Permanente em Saúde (PNEPS), importantes para ampliar o conhecimento dos profissionais e a possibilidade de transformação da realidade. Ressalta-se a falta de diretrizes que sistematizem e avaliem esses momentos educativos, reconhecendo a sua possibilidade de mudança institucional ${ }^{29}$ definitiva não só para a efetivação da $\mathrm{P} 4$, como de outras ações de produção de saúde, em uma perspectiva integral.

\section{CONCLUSÃO E IMPLICAÇÕES PARA A PRÁTICA}

A P4 representa, na APS, uma potencialidade no sentido de reverter um modelo hegemônico de compreensão dos usuários e, mesmo, de alguns profissionais, em relação às formas de cuidar e atender os indivíduos e famílias. Ao voltar-se ao cuidado mais integral, aposta-se na redução da sobremedicalização, dos sobrediagnósticos e dos sobretratamentos, que podem causar danos à saúde dos indivíduos e da população.

Os participantes apresentam alguns desafios para que esta prática se efetive, como a falta de momentos de educação permanente, a revisão das práticas profissionais de saúde e, principalmente, a necessidade de um (re) posicionamento da indústria, do comércio, do marketing e da mídia sobre o significado de saúde. Tudo isso pode contribuir, também, para a mudança de perspectiva do próprio usuário.
Com relação às implicações para a prática, a pesquisa contribui para a revisão dos pontos de vista sobre a ética das profissões da área da saúde e dos prestadores do cuidado, no que se refere ao rastreio, diagnóstico ou tratamento, bem como sobre a responsabilidade de todos os atores envolvidos no processo saúde-doença (incluindo o usuário). Destaca-se a importância de o indivíduo tornar-se copartícipe deste processo sem tornar-se vítima de um sistema lucrativo e indiscriminado.

Ao identificar possibilidades e desafios presentes no cotidiano da equipe para o desenvolvimento da P4, evidenciaram-se os emergentes problemas que se originam com a medicalização social e com a pouca tradição com outras terapêuticas na APS. Isso requer condutas interprofissionais, pautadas na competência cultural, horizontalizada e em habilidades de comunicação, sobretudo entre profissionais e usuários. Assim, é necessário que os profissionais das equipes de saúde, para além dos médicos, incorporem a P4 nas suas práticas. Isso pode iniciar ainda durante a formação profissional, de modo a valorizar e investir na educação interprofissional, aliada aos processos de educação permanente, bem como na avaliação das práticas e modelos de atenção à saúde. Considera-se importante investir nos elementos técnicos, ético-filosóficos e políticos que permeiam a P4, avançando para as relações dos profissionais e para as possibilidades de ações colaborativas e harmônicas em defesa de um cuidado ético e integral.

As limitações deste estudo estão relacionadas ao delineamento de pesquisa na estratégia que adotou um único serviço como cenário, o que não permite generalizações dos seus achados, apesar da sua intensidade e exaustividade. Há de considerarse, também, a necessidade de realizar novas investigações que permitam entender a P4 e suas implicações ao longo do tempo e no cotidiano das equipes.

\section{FINANCIAMENTO}

O presente estudo teve o apoio da Universidade Federal da Fronteira Sul (UFFS), da Fundação de Amparo à Pesquisa e Inovação do Estado de Santa Catarina (FAPESC) e da Universidade do Estado de Santa Catarina (UDESC).

\section{CONTRIBUIÇÕES DOS AUTORES}

Desenho do estudo. Carine Vendruscolo.

Coleta ou produção dos dados. Karina Schopf.

Análise de dados. Daniela Savi Geremia. Aline Lemes de Souza. Lucas Lasta Angonese.

Interpretação dos resultados. Carine Vendruscolo. Clarissa Bohrer da Silva. Daniela Savi Geremia. Aline Lemes de Souza. Lucas Lasta Angonese.

Redação e revisão crítica do manuscrito. Karina Schopf. Carine Vendruscolo. Clarissa Bohrer da Silva. Daniela Savi Geremia. Aline Lemes de Souza. Lucas Lasta Angonese.

Aprovação da versão final do artigo. Karina Schopf. Carine Vendruscolo. Clarissa Bohrer da Silva. Daniela Savi Geremia. Aline Lemes de Souza. Lucas Lasta Angonese. 
Responsabilidade por todos os aspectos do conteúdo e a integridade do artigo publicado. Karina Schopf. Carine Vendruscolo. Clarissa Bohrer da Silva. Daniela Savi Geremia. Aline Lemes de Souza. Lucas Lasta Angonese.

\section{EDITOR ASSOCIADO}

Gerson Luiz Marinho (D)

\section{EDITOR CIENTÍFICO}

Ivone Evangelista Cabral (1)

\section{REFERÊNCIAS}

1. Molster CM, Bowman FL, Bilkey GA, Cho AS, Burns BL et al. The evolution of public health genomics: exploring its past, present, and future. Front Public Health. 2018;6:247. http://dx.doi.org/10.3389/ fpubh.2018.00247.

2. Gérvas J, Oliver LL, Pérez-Fernandez M. Family and Community Medicine and its role in preventing health overuse (preventive, diagnostic, therapeutic and rehabilitative). Cien Saude Colet. 2020;25(4):1233-40. http://dx.doi.org/10.1590/1413-81232020254.30082019. PMid:32267426.

3. Borrozzino NF, Fonseca SC. Prevenção Quaternária: envelhecendo em sociedades medicalizadas [dissertação]. São Paulo: Pontifícia Universidade Católica de São Paulo; 2019.

4. Leavell H, Clark EG. Medicina preventiva. São Paulo: McGraw-Hill; 1976.

5. Jamoulle M, Roland M, Bae J-M, Heleno B, Visentin G, Gusso G, et al. Ethical, pedagogical, socio-political and anthropological implications of quaternary prevention. Rev Med Brux. 2018;39(4):383-93. http:// dx.doi.org/10.5712/rbmfc13(40)1860. PMid:30321004.

6. Illich I. A expropriação da saúde: nêmesis da medicina. $4^{\text {a }}$ ed. São Paulo: Nova Fronteira; 1981.

7. Tesser CD, Dallegrave D. Práticas integrativas e complementares e medicalização social: indefinições, riscos e potências na atenção primária à saúde. Cad Saude Publica. 2020;36(9):e00231519. http:// dx.doi.org/10.1590/0102-311x00231519. PMid:32901667.

8. Martins C, Godycki-Cwirko M, Heleno B, Brodersen J. Quaternary prevention: reviewing the concept. Eur J Gen Pract. 2018;24(1):106-11. http://dx.doi.org/10.1080/13814788.2017.1422177. PMid:29384397.

9. Illich I. Nemesis médica: a expropriação da saúde. Barcelona: Barral Editores; 1975.

10. Coll-Benejam T, Bravo-Toledo R, Marcos-Calvo MP, Astier-Peña MP. Impact of overdiagnosis and overtreatment on the patient, the health system and society. Aten Primaria. 2018;50(2, Suppl 2):86-95. http:// dx.doi.org/10.1016/j.aprim.2018.08.004. PMid:30563626.

11. Attena F. Too much medicine? Scientific and ethical issues from a comparison between two conflicting paradigms. BMC Public Health. 2019 jan 22;19(1):97. http://dx.doi.org/10.1186/s12889-019-6442-9. PMid:30669992.

12. Gross DMP, Camacho ACLF, Lage LR, Daher DV, Mota CP. Prevenção quaternária na gestão da atenção primária à saúde: revisão integrativa. Recife Rev enferm UFPE. 2016;10(Supl 4):3608-19. http://:doi.org/10.5205/ reuol.9681-89824-1-ED.1004sup201613.
13. Cooperrider DL, Whitney D, Stavros JM. Manual da Investigação Apreciativa. Rio de Janeiro: Qualitymark; 2008.

14. Armstrong AJ, Holmes CM, Henning D. A changing world, again. How Appreciative Inquiry can guide our growth. Social Sciences \& Humanities Open.2020;2(1):100038. http://dx.doi.org/10.1016/j.ssaho.2020.100038. PMid:34173485.

15. Arnemann CT, Gastaldo D, Kruse MHL. Pesquisa apreciativa: características, utilização e possibilidades para a área da Saúde no Brasil. Interface (Botucatu). 2018;22(24):121-31. http://dx.doi. org/10.1590/1807-57622016.0763.

16. Minayo MCS. O desafio do conhecimento: pesquisa qualitativa em saúde. 14ª ed. São Paulo: Hucitec; 2014.

17. Norman AH, Hunter DJ, Russell AJ. Vinculando a estratégia preventiva de alto risco ao mercado da indústria biomédica: implicações para a saúde pública. Saude Soc. 2017 set;26(3):638-50. http://dx.doi. org/10.1590/s0104-12902017172682.

18. Peduzzi M, Agreli HF. Trabalho em equipe e prática colaborativa na Atenção Primária à Saúde. Interface (Botucatu). 2018;22(2, supl 2):1525-34. http://dx.doi.org/10.1590/1807-57622017.0827.

19. Ceccim RB. Conexões e fronteiras da interprofissionalidade: forma e formação. Interface (Botucatu). 2018;22(2, supl 2):1739-49. http:// dx.doi.org/10.1590/1807-57622018.0477.

20. Pizzanelli M, Almenas M, Quirós R, Pineda C, Cordero E, Taureaux N et al. Prevenção Quaternária: Ética Médica, Avaliação e Eficiência nos Sistemas de Saúde. Rev Bras Med Fam Comunidade. 2016;11(2):7585. http://dx.doi.org/10.5712/rbmfc11(0)1388.

21. Cardoso RV. Prevenção quaternária: um olhar sobre a medicalização na prática dos médicos de família. Rev Bras Med Fam Comunidade. 2015 jun 24;10(35):1-10. http://dx.doi.org/10.5712/rbmfc10(35)1117.

22. Schopf K, Vendruscolo C, Tesser CD, Zocche DAA, Souza AL. Prevenção quaternária na atenção primária à saúde: uma revisão da literatura. In: Vendruscolo C, Tesser CD, Adamy EK, organizadores. Prevenção Quaternária: proposições para a educação e prática interprofissional na Atenção Primária à Saúde. Porto Alegre: Moriá; 2021. p. 46-66.

23. Almenas M, Hidalgo EC, Pineda CA, Muñoz E, Armadillo MLR, Salvatierra $E$ et al. Prevención cuaternaria: como hacer, como ensiñar. Rev Bras Med Fam Comunidade. 2018 set 18;13(supl 1):69-83. http://dx.doi. org/10.5712/rbmfc13(1)1853.

24. Tesser $\mathrm{CD}$. Por que é importante a prevenção quaternária na prevenção? Rev Saude Publica. 2017 dez 4;51:116. http://dx.doi.org/10.11606/ S1518-8787.2017051000041. PMid:29211199.

25. Jamoulle M, Gomes LF. Prevenção quaternária e limites em medicina Rev Bras Med Fam Comunidade. 2014;9(31):186-91. http://dx.doi. org/10.5712/rbmfc9(31)867.

26. Tesser CD. Cuidado clínico e sobremedicalização na atenção primária à saúde. Trab Educ Saúde. 2019;17(2):e0020537. http://dx.doi. org/10.1590/1981-7746-sol00205.

27. Norman AH, Tesser CD. Quaternary prevention: the basis for its operationalization in the doctor-patient relationship. Rev Bras Med Fam Comunidade. 2015;10(35):1-10. http://dx.doi.org/10.5712/ rbmfc10(35)1011.

28. Santos RABG, Uchôa-Figueiredo LR, Lima LC. Apoio matricial e ações na atenção primária: experiência de profissionais de ESF e Nasf. Saúde Debate. 2017;41(114):694-706. http://dx.doi.org/10.1590/01031104201711402.

29. Silva CBG, Scherer MDA. A implementação da Política Nacional de Educação Permanente em Saúde na visão de atores que a constroem. Interface (Botucatu). 2020;24:e190840. http://dx.doi.org/10.1590/ interface. 190840. 